\title{
Das unterschätzte Risiko - auch viral bedingte Atemwegsinfektionen können tödlich verlaufen
}

\author{
Tobias Welte
}

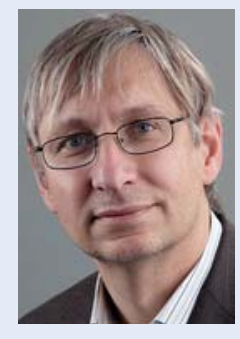

Tobias Welte
Korrespondenzadresse Prof. Dr. Tobias Welte Klinik für Pneumologie Medizinische Hochschule Hannover

Carl-Neuberg-Straße 1 30659 Hannover

welte.tobias@ mh-hannover.de

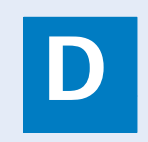

er vergangene Winter war im Hinblick auf viral bedingte Atemwegsinfektionen rekordverdächtig. Am Arbeitsplatz und im Bekanntenkreis blieb kaum jemand von Infekten verschont, die teilweise mit einem hartnäckigen, über Wochen anhaltenden Husten einhergingen. Auch die Zahl der schweren Verläufe war hoch, viele Patienten mussten stationär aufgenommen werden, zeitweise waren Intensivstationen fast vollständig durch Patienten mit respiratorischem Versagen aufgrund von Atemwegsinfektionen belegt. Auffallend war dabei, dass viele der Patienten jung waren und die Erkrankung bei bis dahin vollkommen gesunden Patienten ohne jegliche Ko-Morbidität auftrat. Ein Teil dieser intensivmäßig behandlungsbedürftigen Fälle war Folge einer Influenzainfektion. 2016 trat die Influenzawelle verhältnismäßig spät auf, erste Fälle wurden erst Ende Januar berichtet, der Gipfel der Epidemie lag Ende Februar, es gab jedoch noch Neuerkrankungen bis Ende April. Am Anfang traten fast ausschließlich Influenza AInfektionen (H1N1) auf, im späteren Verlauf kam es auch vereinzelt zu Influenza B-Infektionen. H1N1 scheint gerade bei jungen Menschen mit guter Immunabwehr zu einer überschießenden Immunreaktion zu führen, nicht der Erreger selbst, sondern die durch ihn ausgelöste massive Zytokin- und Mediatorfreisetzung aus Entzündungszellen führt zu Epithelschäden und einem Zusammenbruch der epithelialen Barriere, die sich klinisch im Bild eines foudroyanten ARDS äußert. Allerdings gelang bei Weitem nicht bei allen Patienten mit diesem klinischen Bild der Nachweis von Influenzaviren, auch andere virale Erreger wie humane Metapneumoviren, Parainfluenza-, Adeno- und RS-Viren wurden nachgewiesen, bei einer Reihe von Patienten gelang gar kein Erregernachweis. Zumindest auf unserer Intensivstation gab es jedoch ein Charakteristikum, das auf alle Patienten ausnahmslos zutraf: Keiner der Patienten im respiratorischen Versagen war Influenza-geimpft, auch wenn für einen Teil der Patienten die Impfung in den Richtlinien der Ständigen Impfkommission (STIKO) generell empfohlen wird, wie zum Beispiel für Beschäftigte im
Gesundheitswesen, die bekanntermaßen ein überdurchschnittliches großes Risiko für Influenzainfektionen haben. Obwohl die meisten Krankenhäuser diese Impfung inzwischen kostenlos für alle Mitarbeiter anbieten, wird die Möglichkeit nur selten, in der MHH von knapp 20\% der Mitarbeiter, genutzt. Es bleibt mir ein Rätsel, warum die Angst vor den, meist lokalen und sehr selten schweren, Impfnebenwirkungen so viel größer ist als die Angst vor der nicht ganz seltenen schwer verlaufenden Influenza, selbst bei Mitarbeitern, die junge Menschen an Influenza haben sterben sehen.

Nora Drick [1] beschreibt in ihrem Artikel in dieser Ausgabe von Intensivmedizin up2date alle wesentlichen viralen Atemwegserkrankungen, die zu einem respiratorischen Versagen führen können und erläutert die momentan möglichen diagnostischen und therapeutischen Möglichkeiten. Sie zeigt dabei auf, dass für die meisten Infektionen nur unzureichende virustatische Therapien vorhanden sind, die zudem, wie beispielsweise die Ribavirintherapie bei RS-Virusinfektionen, mit erheblichen Nebenwirkungen verbunden sind. Hier muss intensiv in die zukünftige Medikamentenentwicklung investiert werden, was ohne eine öffentliche Unterstützung von Forschung und Entwicklung nicht erfolgreich ein wird.

Solange kausale Therapiemöglichkeiten nur in Ausnahmefällen zur Verfügung stehen, bleibt nichts anderes übrig als darauf zu hoffen, dass der Mensch selbst mit der Infektion fertig wird und gesundet. Bei respiratorisch insuffizienten Patienten muss dafür die Atmungsfunktion für Tage und manchmal Wochen überbrückt werden. Die Beatmungstherapie hat seit der bahnbrechenden Arbeit des Amerikanischen ARDS Netzwerks [2] zur protektiven drucklimitierten Beatmung mit niedrigen Zugvolumen ( $6 \mathrm{ml} / \mathrm{kg}$ Körpergewicht) große Fortschritte gemacht, ohne dass die Sterblichkeit dieser Patienten wesentlich unter 20\% gesunken wäre. Mit der H1N1-Pandemie der Jahre 2009-2011 sind extrakorporale Lungenersatzverfahren in den Blickpunkt gerückt [3]. Vor allem veno- 
venöse ECMO (extracorporal membran oxigenation)Systeme finden inzwischen in Deutschland breite Anwendung, wie in einer gerade publizierten Analyse von Krankenhausdaten gezeigt wurde [4]. Axel Schmutz und seine Kollegen aus Freiburg zeigen in ihrem Artikel die Möglichkeiten einer ECMO-Behandlung in der modernen Intensivmedizin auf [5]. Sie zeigen jedoch auch, dass es sich bei dieser Behandlung noch immer um ein Verfahren handelt, das viel Erfahrung seitens der Anwender erfordert, weil eine Reihe technischer Schwierigkeiten die Effektivität der ECMOBehandlung einschränken können und weil es sich nach wie vor um ein komplikationsreiches Verfahren handelt, das die Erfahrungen, aber auch technische Möglichkeiten zur Behandlung von Komplikationen erforderlich macht [6]. Nicht der Ehrgeiz, auch in seinem Krankenhaus solche avancierte Medizin durchführen zu können, und auch nicht die Gewinnerwartungen, die durch Abrechnung einer hochpreisigen DRG auf Krankenhausseite oder durch Verkauf der teuren Systeme auf Industrieseite entstehen, sollten über die Anwendung von ECMO bei Patienten entscheiden. Alleine die Kompetenz der Behandler mit dem Verfahren und die Sicherheit in der Beherrschung von Komplikationen sollte als Kriterium für das Einleiten einer ECMO-Therapie gelten.

\section{Literatur}

1 Drick N, Welte T. Intensivpflichtige Virusinfektionen der unteren Atemwege. Intensivmedizin up2date 2016; 12: $159-174$

2 The Acute Respiratory Distress Syndrome Network. Ventilation with lower tidal volumes as compared with traditional tidal volumes for acute lung injury and the acute respiratory distress syndrome. N Engl J Med 2000; 342: 1301 - 1308

3 ANZIC Influenza Investigators. Webb SA, Pettilä V, Seppelt I et al. Critical care services and 2009 H1N1 influenza in Australia and New Zealand. N Engl J Med 2009; 361: 1925-1934

4 Karagiannidis C, Brodie D, Strassmann S et al. Extracorporeal membrane oxygenation: evolving epidemiology and mortality. Intensive Care Med 2016; 42: 889-896

5 Schmutz A, Benk C, Wengenmayer T. Venovenöse extrakorporale Membranoxygenierung. Intensivmedizin up2date 2016; 12: $139-155$

6 lus F, Sommer W, Tudorache I et al. Veno-veno-arterial extracorporeal membrane oxygenation for respiratory failure with severe haemodynamic impairment: technique and early outcomes. Interact Cardiovasc Thorac Surg 2015 ; 20: 761 - 767 